\title{
Erratum to: Fractures and vector valued maps
}

\section{Domenico Mucci}

Published online: 10 November 2009

C) Springer-Verlag 2009

\section{Erratum to: Calc. Var. DOI 10.1007/s00526-004-0282-9}

The isoperimetric inequality of Proposition 3.1 from [1] is false, in general. In fact, its proof contains a mistake, as Eq. (3.4) is false. If e.g. $n=2$, such an isoperimetric inequality is not satisfied if $u \in B V\left(\Omega, \mathbb{R}^{2}\right)$ is not a $W^{1,1}$-function. Taking for example $\Omega=$ $(-1,1) \times(-1,1)$ and $u\left(x_{1}, x_{2}\right)=\left(x_{1}, x_{2}\right)$ if $x_{1}<0$, whereas $u\left(x_{1}, x_{2}\right)=\left(x_{1}+1, x_{2}\right)$ if $x_{1}>0$, we have

$$
\left(\partial G_{u}\right)\left\llcorner\Omega \times \mathbb{R}^{2}=\gamma_{1 \#} I-\gamma_{2 \#} I, \quad I:=\llbracket-1,1 \rrbracket,\right.
$$

where $\gamma_{1}(\lambda):=(0, \lambda, 0, \lambda)$ and $\gamma_{2}(\lambda):=(0, \lambda, 1, \lambda)$, for $-1<\lambda<1$. This example also shows that Eq. (4.4) in Proposition 4.2 from [1] is false, in general, as we have

$$
(\operatorname{Det} \nabla u)^{s}=\frac{1}{2} \mathcal{H}^{1}\left\llcorner J_{u}, \quad J_{u}=\{0\} \times(-1,1) .\right.
$$

As a consequence, it is not possible to deal with singular sets of any dimension lower that $n-1$, and to study the related variational problems as in [1, Sect. 5].

On the other hand, it turns out that the isoperimetric inequality in Proposition 3.1 holds true if one assumes in addition that $u \in W^{1, n-1}\left(\Omega, \mathbb{R}^{n}\right)$. Therefore, using the same techniques as in [1], the $(n-k)$-dimensional fracture $S_{n-k}(u)$ of maps $u$ in $\operatorname{Cf}^{p}\left(\Omega, \mathbb{R}^{N}\right) \cap$ $W^{1, k-1}\left(\Omega, \mathbb{R}^{N}\right)$, see Definition 5.1 , can be defined as in Definition 5.5 , for every integer $2 \leq k \leq \min (n, N)$. Existence results can be then obtained for the minimum of the functional

The online version of the original article can be found under doi:10.1007/s00526-004-0282-9.

D. Mucci $(\bowtie)$

Dipartimento di Matematica dell'Università di Parma, Viale G. P. Usberti 53/A,

43100 Parma, Italy

e-mail: domenico.mucci@unipr.it 


$$
\mathcal{F}_{k}(u):=\int_{\widetilde{\Omega}} \Phi(|\vec{M}(D u)|) d x+\int_{\widetilde{\Omega}}|D u|^{k-1} d x+\mathcal{H}^{n-k}\left(S_{n-k}(u)\right),
$$

for $2 \leq k \leq \min (n, N)$, where $\Phi$ is defined as in Theorems 5.9 and 5.10.

Acknowledgements I thank Duvan Henao and Carlos Mora-Corral for pointing out the above mentioned mistake and counterexample.

\section{Reference}

1. Mucci, D.: Fractures and vector valued maps. Calc. Var. 22, 391-420 (2005) 